\title{
Liquid exfoliation of layered materials
}

\author{
Valeria Nicolosi $^{1,2}$, Manish Chhowalla ${ }^{3}$, Mercouri G. Kanatzidis ${ }^{4}$, Michael S. Strano ${ }^{5}$ \\ and Jonathan N. Coleman ${ }^{1 *}$ \\ ${ }^{1}$ School of Physics and CRANN, Trinity College Dublin, D2, Ireland \\ ${ }^{2}$ School of Chemistry, Trinity College Dublin, D2, Ireland \\ ${ }^{3}$ Materials Science and Engineering, Rutgers University, Piscataway, New Jersey, USA \\ ${ }^{4}$ Department of Chemistry, Northwestern University, Evanston, IL 60208 USA \\ ${ }^{5}$ Department of Chemical Engineering, Massachusetts Institute of Technology, Cambridge, \\ MA 02139 USA \\ *colemaj@tcd.ie
}

\begin{abstract}
The exfoliation of layered crystals is a simple and versatile method for producing atomically thin, 2-dimensional nanosheets from a range of materials such as graphene, $\mathrm{MoS}_{2}$ and $\mathrm{MnO}_{2}$. Such exfoliated nanosheets have many exciting properties that are dramatically different to those of the bulk crystal. As such, these materials will rejuvenate existing applications as well as facilitating new ones. Many future fields of use will require very large quantities of exfoliated nanosheets. The most promising, scalable method for producing high quality nanosheets is exfoliation in a liquid environment. In this review we discuss liquid exfoliation techniques, focusing on potential applications and future directions.
\end{abstract}


In 1824, Thomas H. Webb heated a mineral similar to mica and, by thermal exfoliation, transformed it into what is today a valuable commodity with applications as an ion exchange resin, an insulating material and a structural binder in cement. He named the mineral for its worm-like appearance upon exfoliation as vermiculite (figure 1), from the Latin vermiculare meaning "to breed worms". Almost 200 years later, in 2004, Geim and Novosolov showed that scotch tape could be used to exfoliate graphite into single atomic layers of graphene, demonstrating the thinnest electronic devices in scientific history.(1) As a process, exfoliation of layered solids has had a transformative effect on materials science and technology, creating essentially new materials from those we find around us. With intensified interest in nanotechnology, exfoliation has proven to be a powerful way produce new, low-dimensional materials, unlocking unprecedented properties. This review examines this sometimes understated process and the impact it has had on science and technology.

Layered materials are defined as solids with strong in-plane chemical bonds but weak out of plane, van der Waals bonds. Such materials can be sheared parallel or expanded normal to the in-plane direction. In the extreme limit, these processes lead to exfoliation, yielding nanometer thin, even atomically thin, sheets or nanosheets. A flurry of research activity has highlighted the new and unusual properties of nanosheets that are not at all characteristic of the bulk precursor.

There are many types of layered material,(2) which can be grouped into diverse families (figure 1). The simplest are the atomically thin, hexagonal sheets of graphene( 1 ) and hexagonal boron nitride (hBN).(3) Transition metal dichalcogenides (TMDs - $\mathrm{MoS}_{2}$, WSe $e_{2}$ etc) and metal halides $\left(\mathrm{CdI}_{2}, \mathrm{MgBr}_{2}\right.$ etc) have near-identical structures and consist of a plane of metal atoms sandwiched between planes of halide/chalcogen atoms.(4) Layered metal oxides (e.g. $\mathrm{MnO}_{2}, \mathrm{MoO}_{3}, \mathrm{LaNb}_{2} \mathrm{O}_{7}$ ) and layered double hydroxides (LDHs e.g. $\left.\mathrm{Mg}_{6} \mathrm{Al}_{2}(\mathrm{OH})_{16}\right)$ represent a diverse class of materials with a large variety of structures.(5) Similarly, layered silicates, or clays, are minerals and exist as many different types with well-known examples being montmorillonite or the micas.(6) While many other layered 
materials exist, all share this planar, anisotropic bonding and therefore the potential to be exfoliated into nanosheets.

Many layered materials are themselves interesting, even before exfoliation, due to useful intrinsic properties. For example, TMDs(4) occur as more than 40 different types depending on the combination of chalcogen $(\mathrm{S}, \mathrm{Se}$ or $\mathrm{Te})$ and transition metal(4). Depending on the co-ordination and oxidation state of the metal atoms, or doping of the lattice, TMDs can be metallic, semi-metallic or semiconducting(4). Similarly, the many different types of layered metal oxides have interesting electronic, electrochemical and photonic properties.(5) It is this versatility that makes them potentially useful in many application areas. Such diversity is dramatically enhanced upon exfoliation.

\section{Why exfoliate?}

Historically, the most powerful function of exfoliation has been to enable a material to traverse astounding magnitudes of surface area. The area that a solid surface presents to its environment is perhaps the most significant property that determines its chemical and physical reactivity. The ion exchange ability of minerals like vermiculate to purify water at $1000 \mathrm{meq} / \mathrm{kg}$ depends upon its near $10^{6}$ fold increase in surface area after expansion.(6) In structural mechanics, the thickness of layered fillers, like clay or graphite, determines the strength of composites.(7) When heat alone can exfoliate, the layered material can be used as an intumescent (or thermally expansive) material. Hence, vermiculite and graphite, are used for fire retardation in paints and firestop pillows, as they reduce their density upon heating and produce an ash of low thermal conductivity.

As interest in nanotechnology has intensified in recent decades, another important advantage of exfoliation has become obvious. In a layered crystal, the electronic wavefunction extends in three dimensions. However, after exfoliation electrons are constrained to adopt a two dimensional wavefunction, and therefore a new electronic band structure. Completely new fields have been unlocked after exfoliation to single layers. Hence, graphite becomes a graphene monolayer after exfoliation - a new 2D material with 
enormously high carrier mobility and other exciting properties.(1) Likewise, bulk $\mathrm{MoS}_{2}$ is an indirect band gap semiconductor that becomes a direct gap, fluorescent 2D conductor in the limit of complete exfoliation. The power of exfoliation to transform and redefine materials anew is highly compelling.

\section{Large scale exfoliation in liquids?}

The exfoliation of graphite demonstrated by Geim and Novolosov was achieved essentially by rubbing graphite on a surface.(1) Such mechanical exfoliation remains the source of the highest quality graphene samples available and has resulted in some major advances.(1) However, it suffers from low yield and a production rate that is not technologically scalable in its current form. One possible solution is the exfoliation of layered compounds in liquids to give large quantities of dispersed nanosheets. Liquid exfoliation techniques are versatile methods for obtaining sizable quantities of 2D materials which are cheap, easy and scalable and will allow exfoliated nanosheets to be processed using existing industrial techniques such as reel-to-reel manufacturing, etc. This is a critical advantage that cannot be understated and as a result, we focus this review on liquid exfoliation. Here we briefly outline the four main liquid exfoliation techniques for layered materials (see figure 2 for schematics and figure 3 for examples of exfoliated nanosheets).

One of the oldest methods of exfoliating layered crystals with low reductive potential is oxidation and subsequent dispersion into suitable solvents. The best example is that of graphite(8) where treatment with oxidizers such as sulphuric acid and potassium permanganate results in addition of hydroxyl and epoxide groups to the basal plane. The resulting hydrophillicity allows water intercalation and large scale exfoliation to yield graphene oxide upon ultrasonication. The dispersed flakes are predominantly monolayers, typically $100 \mathrm{~s}$ of nanometers across, and stabilized against reaggregation by a negative surface charge at concentrations of up to $1 \mathrm{mg} / \mathrm{ml}$. While dispersed graphene oxide can be chemically reduced, this leads to aggregation unless surfactant or polymer stabilizers are present. While reduction removes most of the oxides, structural defects remain, rendering the properties of GO significantly different from pristine graphene. 
Layered materials can also strongly adsorb guest molecules into the spacing between layers, creating what are called inclusion complexes. Such intercalation forms the basis of another exfoliation method that is widely applied to layered materials including graphite(9), and $\operatorname{TMDs}(10,11)$. Intercalation, often of ionic species, increases the layer spacing, weakening the interlayer adhesion, and reducing the energy barrier to exfoliation. Intercalants such as n-butyllithium $(10,11)$ or $\operatorname{IBr}(9)$ can transfer charge to the layers, resulting in a further reduction of interlayer binding. Subsequent treatment such as thermal $\operatorname{shock}(9)$ or ultrasonication $(10,11)$ in a liquid completes the exfoliation process. The exfoliated nanosheets can be stabilised electrostatically by a surface charge(11) or by surfactant addition.(9) In the case of $\mathrm{MoS}_{2}$, this method tends to give highly exfoliated nanosheets but has drawbacks associated with its sensitivity to ambient conditions.(10)

The related method of ion exchange takes advantage of the fact that LDHs, clays and some types of metal oxides contain an exchangeable interlayer of cationic counterions.(5) For example, $\mathrm{TiO}_{2}$ layered crystals tend to be negatively charged and so contain ions such as $\mathrm{Cs}^{+}$between the layers to ensure charge neutrality.(5) Such ions can be exchanged for protons by soaking in acidic solutions. The protons can then be exchanged for bulky organic ions (eg tetrabutylammonium cations) leading to significant swelling. Alternatively some clays containing small monovalent ions such as sodium swell by intercalation of water.(6) In general swelling facilitates exfoliation by ultrasonication or shear mixing to give negatively charged nanosheets

A more recent strategy for exfoliation is to expose the layered material to ultrasonic waves in a solvent.(12) Such waves generate cavitation bubbles that collapse into high energy jets, breaking up the layered crystallites and producing exfoliated nanosheets. Modelling has shown that if the surface energy of the solvent is similar to that of the layered material, the energy difference between the exfoliated and reaggregated states will be very small, removing the driving force for re-aggregation.(13) Using solvents such as Nmethyl-pyrrolidone, graphene, BN, TMDs and some TMOs have been exfoliated in this way. $(13,14)$ Similarly, ultrasonication in surfactant or polymer solutions gives nanosheets which are electrostatically or sterically stabilised.(2) 


\section{Pioneers}

The exfoliation of layered compounds is an area of research that dates back several centuries. Modern research probably began with Webb and progressed via the work of chemists such as Schafhaeutl, Brodie and Staudenmaier on the production of graphite intercalation compounds and graphite oxide in the mid $19^{\text {th }}$ century.(15) While these early researchers knew of the laminar nature of graphite, the detailed structure was not known until the 1920s.(13) Early attempts to understand exfoliated layered compounds used transmission electron microscopy (TEM) to image few- and possibly even mono-layer exfoliated graphene oxide as early as 1948. However the limitations of available instrumentation made it impossible to verify if monolayers were really observed. $(15,16)$ The 1960s saw a flurry of activity on the cleavage (often using scotch tape) of TMD crystals to give nanosheets approaching monolayer thickness.(17) This was complemented in 1967 by the demonstration that inorganic layered compounds, in this case vermiculite clay, could be exfoliated in liquids by ion intercalation followed by shear mixing.(18) In 1975 a similar technique was used to exfoliate $\mathrm{TaS}_{2}$, possibly producing monolayers.(19) Subsequently, the availability of cheap ultrasonic agitators provides a more effective energy source for exfoliation, leading to the demonstration of ion intercalation assisted exfoliation of $\mathrm{TaS}_{2}, \mathrm{NbS}_{2}, \mathrm{MoS}_{2}$ as well as layered oxides. $(11,20,21)$ In these reports, TEM and XRD suggested that monolayers had been produced. A decade later, in 2000, the exfoliation of layered double hydroxides by ion exchange followed by stirring or reflux was demonstrated.(22)

Much of this work has historically been limited by the instrumentation available. Early TEM characterisation used techniques such as intensity analysis(20) or shadowing(17) which would not meet today's standards of proof for imaging a single layer. However, the development of scanning tunnelling microscopy (STM) and atomic force microscopy (AFM) in the 1980s and recent advances in scanning transmission electron microscopy have changed this entirely. By the time Geim et al produced nanosheets by mechanical exfoliation,(1) a new generation of scientific instrumentation with atomic scale 
resolution had emerged to fully analyse exfoliated materials with unprecedented precision. The result has been a renewed surge in interest in these exotic, molecular scale materials.

\section{Recent advances in liquid exfoliation}

Although the exfoliation of layered materials has been an on-going field over the last few decades, the rise of graphene has stimulated renewed interest in this area. For this reason we outline the recent developments in the liquid exfoliation of layered crystals taking Geim and Novoselov's 2004 paper as a starting point.(1)

In 2006, Ruoff et al demonstrated platelets of graphite oxide with monolayer thickness.(8) Now known as graphene oxide (GO), this material is electrically insulating, although it can be rendered conducting via reduction.(8) The oxidation allows one to control the amount and type of attached oxides potentially enabling control of electrical conductivity and luminescence.(23) A disadvantage of this method is that it necessarily introduces chemical groups and defects that scatter electrons, giving relatively high resistivity. It should be noted that graphene oxide exfoliation has been the subject of many papers and reviews.(8)

Around the time of Geim's seminal paper, McEwan et al. produced good quality nano-graphite by sonicating graphite in solvents.(24) However, pristine, non-covalently functionalized graphene was not produced by liquid exfoliation until 2008.(12, 13) By ultrasonicating graphite in solvents with surface tensions of $\sim 40 \mathrm{~mJ} / \mathrm{m}^{2}$, low concentration dispersions of small but high-quality graphene flakes were demonstrated. Recent improvements have substantially enhanced both the dispersed concentration $(\sim 30 \mathrm{mg} / \mathrm{ml})$ and nanosheet size (up to $5 \mu \mathrm{m}$ ).(13) This method is promising for applications where good electrical performance is required. Recent results on printed electronic circuits have yielded mobility values of $\sim 100 \mathrm{~cm}^{2} / \mathrm{Vs},(25)$ some of the highest for any chemically exfoliated graphenes. In addition, liquid exfoliation of graphene with minimal lattice defects allows effective reinforcement of polymers.(7) 
Similar methods have recently been used to exfoliate both $\mathrm{h}-\mathrm{BN}(2,3,14)$ and a range of TMDs in both solvents and aqueous surfactant solutions.(14, 26) Dispersions of TMDs such as $\mathrm{MoS}_{2}, \mathrm{WS}_{2}, \mathrm{MoSe}_{2}, \mathrm{MoTe}_{2}, \mathrm{TaSe}_{2}$ and $\mathrm{NbSe}_{2},(26)$ were obtained at concentrations as high as $40 \mathrm{mg} / \mathrm{ml}$.(2) As with graphene, dispersion works for solvents or solvent blends(27) with surface energies which minimise the energetic cost of exfoliation.(2) TMDs prepared by this scalable method have already attracted considerable interest as composite fillers(28) and for energy storage.(29) For example, Smith et al. have demonstrated solution processed $\mathrm{MoS}_{2} /$ nanotube composite cathodes for $\mathrm{Li}$ ion battery's with reversible capacity of $>200 \mathrm{mAhg}^{-1}$.(26) Chemical pre-treatment also facilitates exfoliation by sonication in solvents. For example, functionalisation allows the exfoliation of LDHs in alcohols(30) while acid treatment of metal aluminium carbides/nitrides gives metal carbide nanosheets with fluorinated or hydroxylated surfaces that can be exfoliated in methanol.(31)

A number of advances have been made in the exfoliation of layered materials via ion intercalation. Strano et al. have demonstrated that dispersions of predominately bi- and tri-layer graphene can be produced from graphite intercalation compounds by thermal expansion followed by sonication in surfactant solution.(9) Eda et al have used ion intercalation to exfoliate $\mathrm{MoS}_{2},(10)$ obtaining photoluminescent monolayers. Recently, Zeng et al. demonstrated electrochemical lithium insertion followed by exfoliation for $\mathrm{MoS}_{2}, \mathrm{WS}_{2}, \mathrm{TiS}_{2}, \mathrm{TaS}_{2}, \mathrm{ZrS}$ and graphite.(32). The resultant nanosheets were fabricated into transistors and sensors. Recently, Xiao et al.(28) reported using exfoliated $\mathrm{MoS}_{2}$ to prepare $\mathrm{MoS}_{2}$ /polyethylene oxide nanocomposites as battery electrodes with greatly improved lithiation capacity and cycling behavior. Du et al(33) prepared $\mathrm{MoS}_{2}$ anode electrodes by exfoliation and restacking process, improving stability and achieving capacity as high as $800 \mathrm{mAhg}^{-1}$. Additionally, exfoliation by ion intercalation has been demonstrated for V-VI layered compounds such as $\mathrm{Bi}_{2} \mathrm{Te}_{3}$ and $\mathrm{Bi}_{2} \mathrm{Se}_{3}$.(34)

Of particular interest are layered metal oxides, which are typically exfoliated by ion exchange followed by agitation.(5) Exfoliated oxide nanosheets are most often wide bandgap semiconductors, making them promising for applications such as photocatalysts or 
high-dielectric constant materials.(5, 35) Alternatively, $\mathrm{MnO}_{2}$ and $\mathrm{RuO}_{2}$ nanosheets are either redox-active or semimetallic $(5,35)$ and have demonstrated high performance as electrochemical supercapacitor and battery materials. $(5,35)$ Colloidal dispersions of exfoliated $\mathrm{Cs}_{4} \mathrm{~W}_{11} \mathrm{O}_{36}{ }^{2-}$ nanosheets are photochromic and superior to commonly used materials such as $\mathrm{WO}_{3}$.(5) Interestingly, $\mathrm{MnO}_{2}$ has also been exfoliated by ultrasonication in aqueous surfactant solutions,(26) suggesting that this facile route can be applied to other layered oxides.

Intercalated layered double hydroxides have historically found many applications from catalysis to drug delivery.(5) A successful delamination process was first performed by Adachi-Pagano et al. by exchanging the interlayer anions with dodecylsulfate under reflux in butanol.(36) Recent studies have focused on exfoliating such ion-exchanged materials in solvents such as fomamide(36) and water.(37) High concentration ( $40 \mathrm{~g} / \mathrm{L})$ dispersions of mono- and few-layer LDHs were reported by $\mathrm{Wu}$ et al.(38) Liquid exfoliated LDHs have found applications in areas as diverse as drug delivery and water treatment.(5)

What can liquid exfoliated nanosheets do?

Liquid exfoliation represents a versatile, scalable, and sustainable route for production of 2D nanosheets. In addition, access to suspensions of nanosheets permits processing in ways that would otherwise be difficult or impossible. For example, processing from liquids allows the deposition of individual nanosheets on surfaces, the formation of thin or free standing films, facilitates mixing with other nanomaterials to form heterostructured solids and enables insertion, as fillers, into polymer matrices (figure 4). Such processability opens up many possibilities for use of nanosheets in applications.

The ability to invoke different phases of $2 \mathrm{D}$ materials via tuning the exfoliation chemistry opens up exciting range of possibilities. For example, exfoliation via electrochemical ion intercalation leads to realization of the metallic (1T) phase in layered transition metal chalcogenides that cannot be accessed through other synthesis techniques.(39) Some obvious applications include electrodes for energy storage devices, 
high performance fillers for polymer based composites, electro- and photo-catalysis for hydrogen evolution, nano- and large-area electronics and opto-electronics, and as low friction additives in lubrication systems.

The production of inexpensive 2D nanosheets holds promise for multi-functional polymer composites. Polymer-clay composites can display improved mechanical and barrier properties, usually at loading levels from a few percent to a few tens of percent.(40) Due to the potentially high aspect ratio of exfoliated graphene, polymers loaded with graphene display reinforcement(7), conductivity enhancement(41) and barrier properties(42) at loading fractions below 1vol\%. Solvent processing of $2 \mathrm{D}$ nanosheets makes composite fabrication straightforward and will extend the suite of $2 \mathrm{D}$ fillers beyond clays and graphene.(3, 14) Liquid exfoliation also allows the formation of unusual composites consisting of mixtures of nanosheets, nanotubes and other nano-structures (figure 4).(14, 26) Thus results in synergetic effects - for example, composites of $\mathrm{WS}_{2}$ and carbon nanotubes display very high conductivity but retain much of the character of $\mathrm{WS}_{2}$ films.(14, 26) Such composites are promising for applications in photovoltaic or thermoelectric devices and as electrodes for supercapacitors or batteries.

Supercapacitor electrodes formed from chemically exfoliated graphene nanosheets have yielded energy densities of $20 \mathrm{Whkg}^{-1}$ for packaged devices, approaching those of lead-acid batteries.(43) Recent results on restacked nanosheets films indicate that they maintain their structural integrity upon intercalation and de-intercalation.(26) The wide range of $2 \mathrm{D}$ materials available with varying electrochemical properties, makes them ideal for energy storage. Initial measurements indicate that their capacitive capability is very high, though issues relating to substantial loss after the first few cycles remain. Choosing the correct combinations of materials will allow multiple properties (e.g. surface area, conductivity, electrochemical stability) to be simultaneously improved so as to provide flexibility for designing electrodes for batteries and supercapacitors. For example, materials such as $\mathrm{VS}_{2}, \mathrm{MoO}_{3}$ and $\mathrm{MnO}_{2}$, could be useful for efficient energy storage. 
The possibility that layered materials could catalyse the production of hydrogen from water, for use as a fuel, is a critically important application. The edge molybdenum sites on TMDs are highly active for hydrogen evolution reactions and thus are being considered as electrodes for water splitting using sunlight. With ion exfoliated 2D TMDs, the presence of a metallic (1T) phase makes the entire basal plane catalytically active leading to an improvement in the catalytic activity and a substantial increase in the number of active catalytic sites.(44) Oxide nanosheets (e.g. $\mathrm{TiO}_{2}, \mathrm{MoO}_{3}$ ) are also candidates for hydrogen evolution catalysts.(45)

Liquid phase exfoliation facilitates the integration of the materials into large area electronics through the well-known solution based techniques such as inkjet printing and roll-to-roll coating. Recent results have indicated that the electronic structure of the 2D nanosheets is largely preserved after liquid exfoliation so that fundamental processes can be observed in individual sheets. For example, because exfoliated oxides retain their excellent dielectric properties they can be used as high-k dielectrics in nano-electronics.(35) Alternatively, stacking of different layered perovskites allows the fabrication of artificial multi-ferroic materials for use in future oxide-based electronics and memory devices. Exfoliated oxide nanosheets may also be used in more complex nanodevices such as photoconducting cells, $\mathrm{p}-\mathrm{n}$ junctions and field-effect transistors.(5, 35) Alternatively, $\mathrm{MoS}_{2}$ shows exceptionally stable transistor operation under very large mechanical deformation.(46) The relatively undisturbed electronic structure coupled with flexibility makes $\mathrm{MoS}_{2}$ and other 2D materials useful for next generation large-area electronics and opto-electronics on plastic and paper platforms. In particular, large area, solution cast, TMD thin film transistors with mobility of $\sim 10-50 \mathrm{~cm}^{2} / \mathrm{Vs}$ could replace doped oxide semiconductors for back-plane switching in flexible and transparent displays.

More exotic applications of layered 2D materials will arise from stacking and seamless integration of specific combination of materials to access fundamental properties such as superconductivity and condensates.(47) The integration could be in the vertical or lateral direction so as to allow for different device concepts. The ability to access metallic and semiconducting phases in 2D materials with electrochemical exfoliation also provides 
opportunities for designing molecular scale heterostructures with atomically coherent junctions.(48) These types of superlattice structures could be easily synthesized from liquid dispersions.

\section{Outlook}

It is clear that we are at a pivotal moment in our understanding and control of layered materials. Our ability to employ straightforward techniques to exfoliate them points to a productive future in this area. However, a number of significant challenges remain. In the case of ultrasonication-assisted solvent exfoliation, it will be necessary to develop methods both to monitor and increase the monomer yield and lateral flake size. For ion intercalation based methods, the time required to achieve exfoliation must be reduced. Crucially, the scaleup of liquid exfoliation must be demonstrated. An achievable early target would be a production rate of grams of exfoliated nanosheets per hour.

It will be important to demonstrate exfoliation of a wider range of layered materials. There are many materials offering a rich set of unique features which have not yet been exfoliated but should be amenable to the methods described above. For example, as layered GaSe and InSe are photoluminescent, liquid exfoliation could lead to next generation solution-processed, photostable light emitting diodes. Alternatively, V-VI layered compounds $\left(\mathrm{Bi}_{2} \mathrm{Te}_{3}, \mathrm{Bi}_{2} \mathrm{Se}_{3}\right.$ etc) could form the basis of solution processed thermoelectric materials. $(14,26)$

In addition, exfoliation in liquids will be a good starting point for subsequent chemical modification of nanosheets. For example, chemical functionalisation will allow controlled modification of the properties of exfoliated nanosheets for applications in composites, targeted drug delivery or sensing. It will be critical to develop the chemistry required to attach a wide range of chemical groups to both nanosheet basal plane and edge in a controlled way. 
As exfoliation methods increase in their sophistication and effectiveness, layered materials will yield a host of new 2D systems to explore. The diverse range of properties of known layered crystals means that their exfoliated counterparts will be equally diverse, leading to applications in electronics, photonics, energy storage, structural composites, drug delivery, barrier layers and coatings to name but a few. We envisage exfoliated nanosheets becoming central to nanotechnology in the $21^{\text {st }}$ century. 
Figure captions
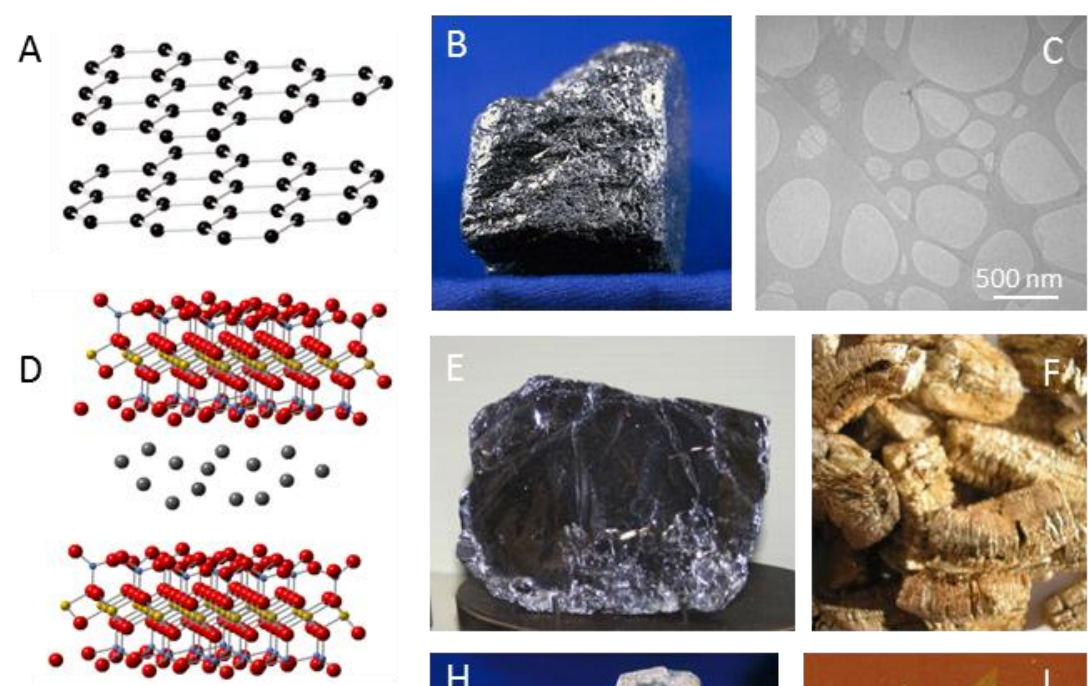

G
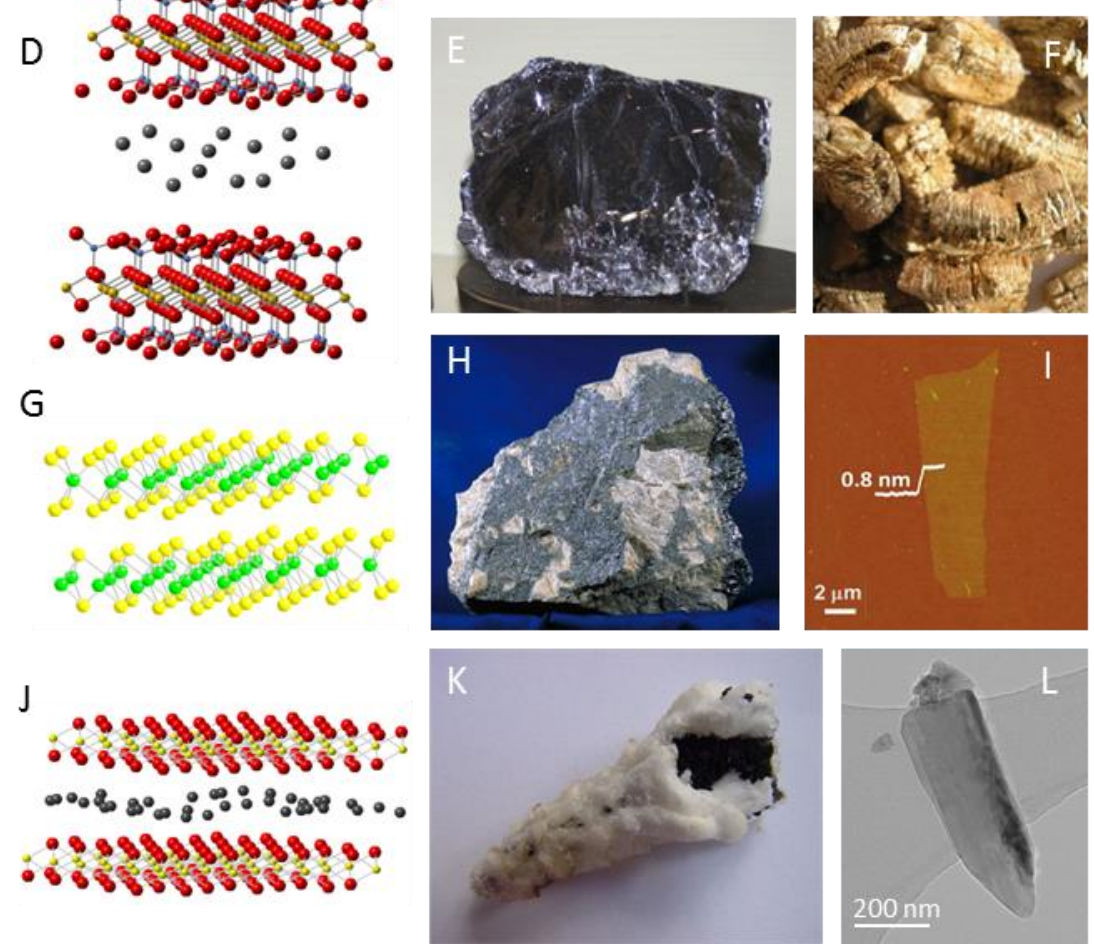

Figure 1: Crystal structures, naturally occurring forms and exfoliated products for four example layered materials. A) Graphite consists of alternating stacks of hexagonally arranged carbon atoms, is a naturally occurring mineral (B) and exfoliates (C) to single atomic layers of carbon called graphene. D) Vermiculite is a layered silicate hydrate which is found naturally as a mineral (E) and can be exfoliated e.g. upon heating (F). G) $\mathrm{MoS}_{2}$ is a layered arrangement of $\mathrm{Mo}$ and $\mathrm{S}$ atoms which is found naturally as the mineral molybdenite $(\mathrm{H})$ and can be exfoliated to $\mathrm{MoS}_{2}$ monolayers (I). J) Layered manganese dioxide occurs naturally as Birnessite $(\mathrm{K})$ and can be exfoliated to give $\mathrm{MnO}_{2}$ nanosheets (L). I and L adapted from (49) and (26) respectively. The layer spacings for each material are: graphite $-0.35 \mathrm{~nm}$, vermiculite $-1.5 \mathrm{~nm}, \mathrm{MoS}_{2}-0.6 \mathrm{~nm}, \mathrm{MnO}_{2}-0.45 \mathrm{~nm}$. 
A

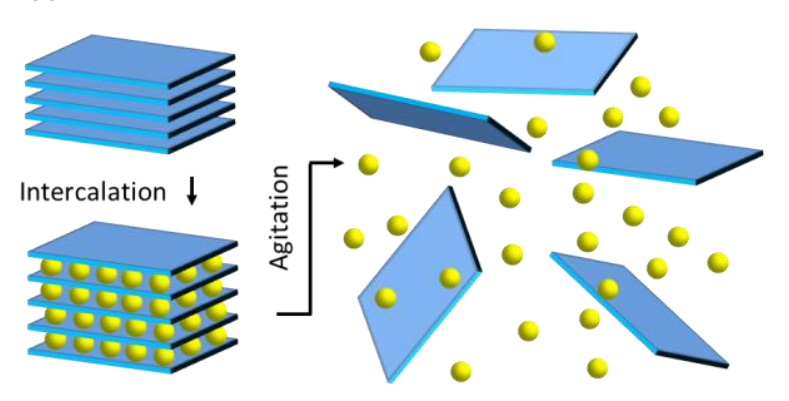

C
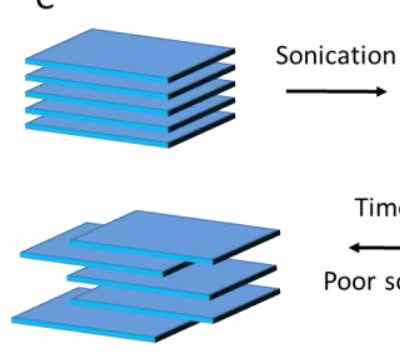

B
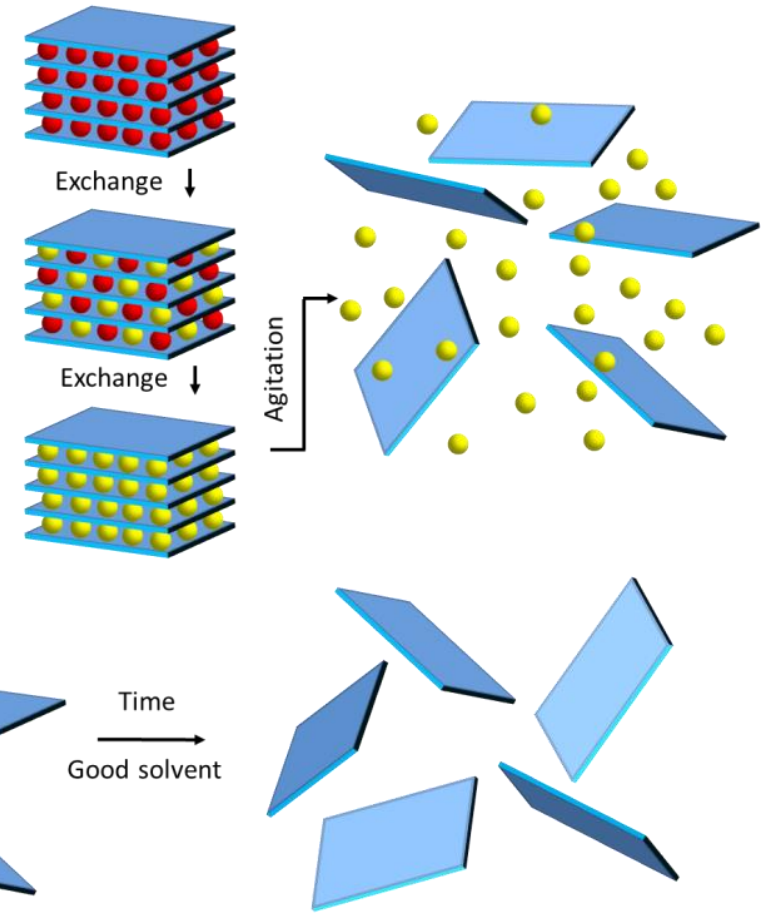

Figure 2: Schematic description of the main liquid exfoliation mechanisms. A) Ion intercalation. Here ions (yellow spheres) are intercalated between the layers in a liquid environment, swelling the crystal and weakening the interlayer attraction. Then, agitation (shear, ultrasonication, thermal etc) can completely separate the layers resulting in an exfoliated dispersion. B) Ion exchange. Some layered compounds contain ions between the layers to balance surface charge on the layers. These ions (red spheres) can be exchanged in a liquid environment for other, often larger ions (yellow spheres). As above, agitation results in an exfoliated dispersion. C) Sonication assisted exfoliation. Here the layered crystal is sonicated in a solvent resulting in exfoliation and nanosheet formation. In "good" solvents, i.e. those with appropriate surface energy, the exfoliated nanosheets are stabilised against reaggregation. Otherwise, for "bad" solvents, reaggregation and sedimentation will occur. This mechanism also describes the dispersion of graphene oxide in polar solvents such as water. NB, solvent molecules are not shown in this figure. 

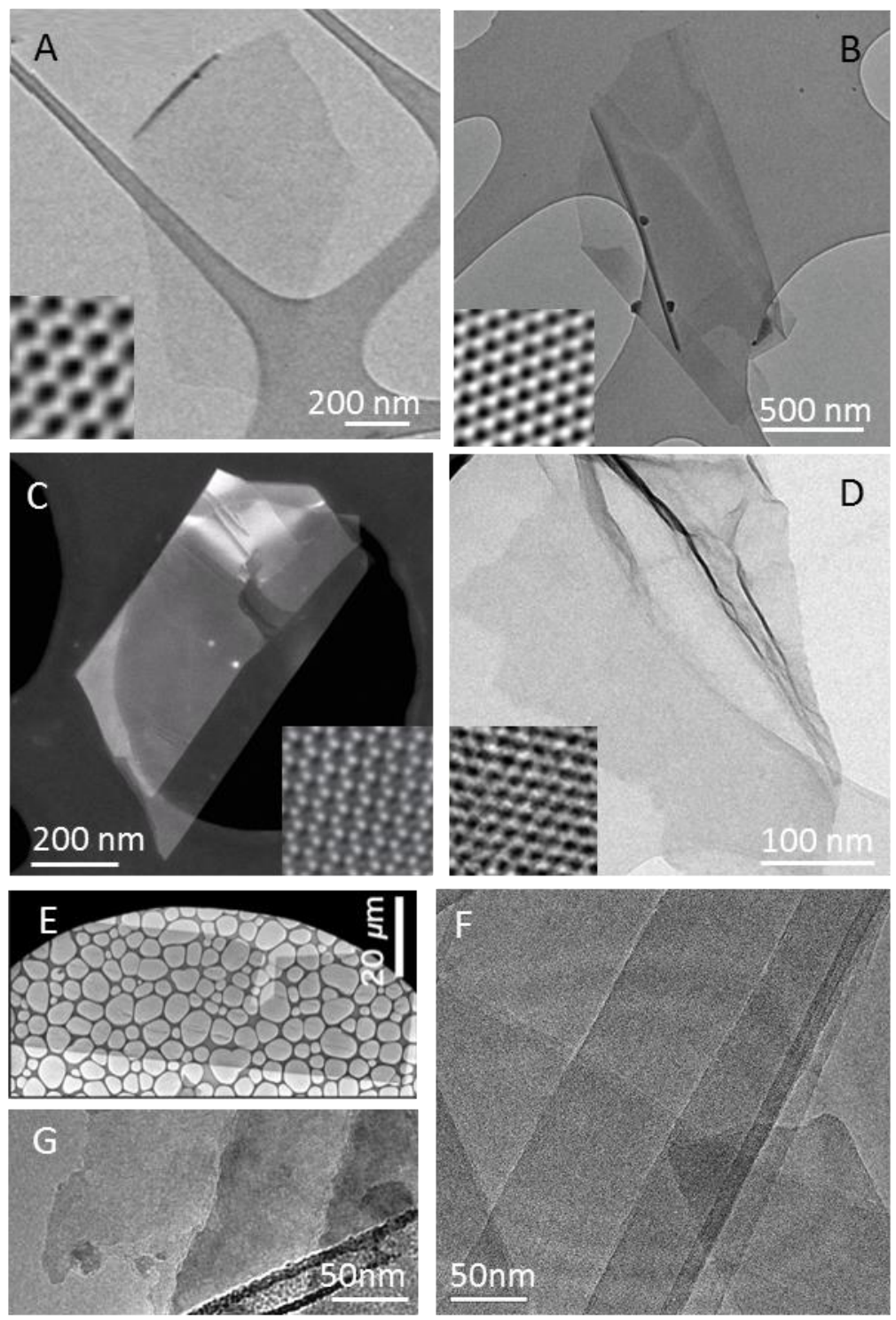

Figure 3: TEM images of liquid exfoliated nanosheets. A) A graphene nanosheet exfoliated by sonication in the solvent N-methyl-pyrrolidone.(12) B) A BN nanosheet exfoliated by sonication in the solvent isopropanol(14). C) $\mathrm{An} \mathrm{MoS}_{2}$ nanosheet exfoliated by sonication in an aqueous surfactant solution(26). D) An $\mathrm{MoS}_{2}$ nanosheet exfoliated by lithium ion intercalation(10). E) A $\mathrm{TiO}_{2}$ nanosheet exfoliated by ion exchange(5). F: Functionalised layered double hydroxide nanosheets exfoliated by sonication in ethanol.(30) G: Hydroxylated metal carbide nanosheets exfoliated by sonication in methanol.(31) Where 
available, high resolution images have been included as insets. All images were adapted from the sources as referenced.
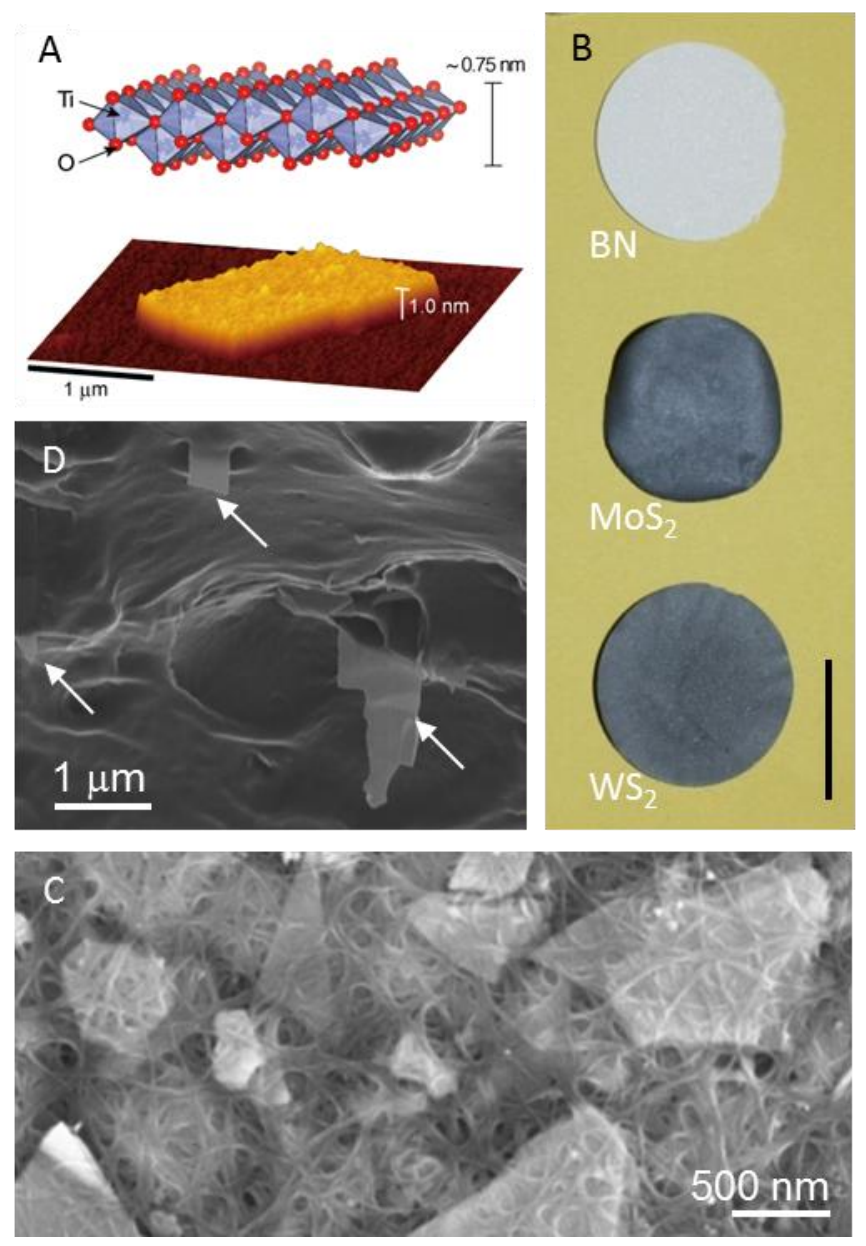

Figure 4: Once layered materials have been exfoliated in liquids, they can be easily processed into a range of structures. A) Titanium dioxide $\left(\mathrm{TiO}_{2}\right)$ nanosheets deposited onto a substrate and imaged by AFM.(35) B) Solution processed free standing films of randomly arranged nanosheets of $\mathrm{BN}, \mathrm{MoS}_{2}$ and $\mathrm{WS}_{2}$ (scale bar $25 \mathrm{~mm}$ ).(14) C) A composite film of carbon nanotubes in a matrix of randomly arranged $\mathrm{WS}_{2}$ nanosheets.(14) D) Solvent exfoliated graphene nanosheets (arrows) embedded in a polymer matrix.(7) All images were adapted from the sources as referenced. 


\section{References}

1. A. K. Geim, Science 324, 1530 (Jun, 2009).

2. See supporting online material for a referenced table of layered materials including structure, applications and exfoliation techniques.

3. D. Golberg et al., Acs Nano 4, 2979 (Jun, 2010).

4. J. A. Wilson, A. D. Yoffe, Advances in Physics 18, 193 (1969).

5. $\quad$ R. Z. Ma, T. Sasaki, Advanced Materials 22, 5082 (Dec, 2010).

6. P. F. Luckham, S. Rossi, Advances in Colloid and Interface Science 82, 43 (Oct, 1999).

7. P. May, U. Khan, A. O'Neill, J. N. Coleman, Journal of Materials Chemistry 22, 1278 (2012).

8. D. R. Dreyer, S. Park, C. W. Bielawski, R. S. Ruoff, Chemical Society Reviews 39, 228 (2010).

9. C. J. Shih et al., Nature Nanotechnology 6, 439 (Jul, 2011).

10. G. Eda et al., Nano Letters 11, 5111 (Dec, 2011).

11. P. Joensen, R. F. Frindt, S. R. Morrison, Materials Research Bulletin 21, 457 (Apr, 1986).

12. Y. Hernandez et al., Nature Nanotechnology 3, 563 (Sep, 2008).

13. J. N. Coleman, Accounts of Chemical Research DOI: 10.1021/ar300009f, (2012).

14. J. N. Coleman et al., Science 331, 568 (Feb, 2011).

15. D. R. Dreyer, R. S. Ruoff, C. W. Bielawski, Angewandte Chemie-International Edition 49, 9336 (2010).

16. G. Ruess, F. Vogt, Monatshefte Fur Chemie 78, 222 (1948).

17. R. F. Frindt, Journal of Applied Physics 37, 1928 (1966).

18. G. F. Walker, W. G. Garrett, Science 156, 385 (1967).

19. D. W. Murphy, G. W. Hull, Journal of Chemical Physics 62, 973 (1975).

20. C. Liu, O. Singh, P. Joensen, A. E. Curzon, R. F. Frindt, Thin Solid Films 113, 165 (1984).

21. M. M. J. Treacy, S. B. Rice, A. J. Jacobson, J. T. Lewandowski, Chemistry of Materials 2, 279 (May-Jun, 1990).

22. M. Adachi-Pagano, C. Forano, J. P. Besse, Chemical Communications, 91 (2000).

23. G. Eda, M. Chhowalla, Advanced Materials 22, 2392 (Jun, 2010).

24. J. S. Bunch, Y. Yaish, M. Brink, K. Bolotin, P. L. McEuen, Nano Letters 5, 287 (Feb, 2005).

25. F. Torrisi et al., Acs Nano 6, 2992 (Apr, 2012).

26. R. J. Smith et al., Advanced Materials 23, 3944 (Sep, 2011).

27. K. G. Zhou, N. N. Mao, H. X. Wang, Y. Peng, H. L. Zhang, Angewandte ChemieInternational Edition 50, 10839 (2011).

28. J. Xiao et al., Chemistry of Materials 22, 4522 (Aug, 2010).

29. Y. L. Liang et al., Advanced Materials 23, 640 (Feb, 2011).

30. Y. Xu, K. Kominami, Y. Ishikawa, Q. Feng, Journal of Colloid and Interface Science 386, 107 (2012).

31. M. Naguib et al., Acs Nano 6, 1322 (Feb, 2012).

32. Z. Y. Zeng et al., Angewandte Chemie-International Edition 50, 11093 (2011).

33. G. D. Du et al., Chemical Communications 46, 1106 (2010).

34. Z. F. Ding et al., Journal of Materials Chemistry 19, 2588 (2009).

35. M. Osada, T. Sasaki, Advanced Materials 24, 210 (Jan, 2012).

36. R. Z. Ma, Z. P. Liu, L. Li, N. Iyi, T. Sasaki, Journal of Materials Chemistry 16, 3809 (2006).

37. T. Hibino, M. Kobayashi, Journal of Materials Chemistry 15, 653 (2005). 
38. Q. L. Wu, A. Olafsen, O. B. Vistad, J. Roots, P. Norby, Journal of Materials Chemistry 15, 4695 (2005).

39. J. Heising, M. G. Kanatzidis, Journal of the American Chemical Society 121, 638 (Feb, 1999).

40. S. Pavlidou, C. D. Papaspyrides, Progress in Polymer Science 33, 1119 (Dec, 2008).

41. S. Stankovich et al., Nature 442, 282 (Jul, 2006).

42. H. D. Huang et al., Journal of Membrane Science 409, 156 (Aug, 2012).

43. $\quad$ Y. W. Zhu et al., Science 332, 1537 (Jun, 2011).

44. A. B. Laursen, S. Kegnaes, S. Dahl, I. Chorkendorff, Energy \& Environmental Science 5, 5577 (Feb, 2012).

45. A. Kudo, Y. Miseki, Chemical Society Reviews 38, 253 (2009).

46. J. Pu et al., Nano Letters 12, 4013 (2012/08/08, 2012).

47. K. S. Novoselov, A. H. C. Neto, Physica Scripta T146, (Jan, 2012).

48. G. Eda et al., Acs Nano 6, 7311 (2012/08/28, 2012).

49. Z. Y. Yin et al., Acs Nano 6, 74 (Jan, 2012). 